\title{
Stretchable, self-healing, transient macromolecular elastomeric gel for wearable electronics
}

Mingming Hao ${ }^{1,2}$, Lianhui $\mathrm{Li}^{1}$, Shuqi Wang ${ }^{1}$, Fuqin Sun ${ }^{1}$, Yuanyuan Bai ${ }^{1}$, Zhiguang Cao ${ }^{1}$, Chunyan $\mathrm{Qu}^{1,2}$ and Ting Zhang ${ }^{1}$

\begin{abstract}
Flexible and stretchable electronics are emerging in mainstream technologies and represent promising directions for future lifestyles. Multifunctional stretchable materials with a self-healing ability to resist mechanical damage are highly desirable but remain challenging to create. Here, we report a stretchable macromolecular elastomeric gel with the unique abilities of not only self-healing but also transient properties at room temperature. By inserting small molecule glycerol into hydroxyethylcellulose (HEC), forming a glycerol/hydroxyethylcellulose (GHEC) macromolecular elastomeric gel, dynamic hydrogen bonds occur between the HEC chain and the guest small glycerol molecules, which endows the GHEC with an excellent stretchability (304\%) and a self-healing ability under ambient conditions. Additionally, the GHEC elastomeric gel is completely water-soluble, and its degradation rate can be tuned by adjusting the HEC molecular weight and the ratio of the HEC to glycerol. We demonstrate several flexible and stretchable electronics devices, such as self-healing conductors, transient transistors, and electronic skins for robots based on the GHEC elastomeric gel to illustrate its multiple functions.
\end{abstract}

\section{Introduction}

Flexible and stretchable electronics are used extensively in human-activity monitoring ${ }^{1-5}$, personal healthcare ${ }^{3,6-9}$, and human-computer interactions ${ }^{10-13}$, since their unique characteristics, such as having a low modulus, being lightweight, and having a high flexibility and stretchability, allow for the conformal integration of electronics with nonplanar surfaces ${ }^{3,14}$. However, such flexible and stretchable electronics are still susceptible to tearing, puncturing and other forms of mechanical failure, leading to a loss in electrical performance, which greatly limits their further application. Encapsulation is an effective way to protect these electronics, but sacrifices its

\footnotetext{
Correspondence: Ting Zhang (tzhang2009@sinano.ac.cn)

${ }^{1} \mathrm{i}$-Lab, Key Laboratory of multifunctional nanomaterials and smart systems,

Suzhou Institute of Nano-tech and Nano-bionics, Chinese Academy of

Sciences, 215123 Suzhou, China

${ }^{2}$ Nano Science and Technology Institute, University of Science and Technology

of China, 96 Jinzhai Road, 230026 Hefei, Anhui, China

These authors contributed equally: Mingming Hao, Lianhui Li
}

stretchability, lightness, and thickness. Endowing flexible and stretchable electronics with a self-healing ability to resist mechanical damage is insistently demanded for future flexible and stretchable electronics ${ }^{15}$. Moreover, with the rapid development of flexible and stretchable electronics, the prevention of sensitive data leakage, the reduction of electronic waste, and biomedical implants without a second surgery are gradually becoming new requirements for their applications. Transient electronic devices that have the ability to disappear in a controlled manner are also extremely desirable.

In recent years, remarkable advances in self-healing materials ${ }^{16-20}$ and transient electronics ${ }^{21-26}$ have been made. For example, a healable thermoplastic elastomer material based on the use of supramolecular interactions for spontaneous healing was reported by Leibler, which can structurally heal after more than 1 week at $23^{\circ} \mathrm{C}{ }^{20}$. Recently, Bao and colleagues demonstrated an intrinsically self-healable composite conductor composed of a

\section{(c) The Author(s) 2019}

(c) (i) Open Access This article is licensed under a Creative Commons Attribution 4.0 International License, which permits use, sharing, adaptation, distribution and reproduction cc) in any medium or format, as long as you give appropriate credit to the original author(s) and the source, provide a link to the Creative Commons license, and indicate if changes were made. The images or other third party material in this article are included in the article's Creative Commons license, unless indicated otherwise in a credit line to the material. If material is not included in the article's Creative Commons license and your intended use is not permitted by statutory regulation or exceeds the permitted use, you will need to obtain permission directly from the copyright holder. To view a copy of this license, visit http://creativecommons.org/licenses/by/4.0/. 
supramolecular polymer (made from dimer acid, triamine and urea) and nanostructured nickel microparticles. The composite showed a good self-healing ability and stretchability (approximately $35 \%)^{17}$. In the field of transient electronics, $\mathrm{Yu}$ and coworkers reported a moisturetriggered physically transient electronic that was composed of functional devices on top of hydrolytically liable polyanhydride substrates. However, it takes dozens of hours for the transient electronics to degrade ${ }^{24}$. To the best of our knowledge, despite their rapid development, flexible electronics based on multifunctional materials that are simultaneously stretchable, self-healing and have transient properties have not yet been reported.

Here we report a unique macromolecular elastomeric gel with the abilities of stretchability, self-healing properties and transient properties at room temperature (Fig. 1). The multifunctional elastomeric gel is fabricated by pouring an aqueous solution of a glycerol and hydroxyethylcellulose mixture. Folded hydroxyethylcellulose (HEC) molecule chains and the reversible formation of the macromolecular and the small molecule hydrogenbonding networks are introduced by the abundant hydroxy groups, providing the GHEC with an excellent stretchability and self-healing function. The GHECs show a superior stretchability (over 304\% strain $(\varepsilon)$ ) and a fast moisture-triggered self-healing time $(\sim 11 \mathrm{~min})$ at room temperature $\left(25^{\circ} \mathrm{C}\right)$ and ambient humidity $(40 \% \mathrm{RH})$. Additionally, the GHEC also exhibits a controllable transient behavior, which is attributed to the solubility of GHEC in water. By varying the ratio of glycerol to HEC in the GHEC, the desired time scales for the transience range from minutes to hours. Moreover, a series of flexible and stretchable electronics devices, such as self-healing conductors, transient transistors, and electronic skins for robots, are designed and fabricated to demonstrate the aforementioned multiple functions.

\section{Materials and methods \\ Materials}

Hydroxyethylcellulose (HEC, 200, 2000, and $5000 \mathrm{mPa} . \mathrm{s}$, $25^{\circ} \mathrm{C}$ ) was purchased from Shanghai Aladdin Industrial Corporation Co. Ltd. Glycerol (analytical grade) was purchased from Shanghai Sinopharm Chemical Reagent Co. Ltd. Silver nanowires (AgNWs) $(0.05 \mathrm{wt} \%$ purity, diameter: $15-20 \mathrm{~nm}$, and length: $30-40 \mu \mathrm{m})$ and singlewalled carbon nanotubes (SWCNTs) $>98 \mathrm{wt} \%$, diameter: $5-8 \mathrm{~nm}$, and length: $0.5-5 \mu \mathrm{m})$ were purchased from Nanjing XFNANO Materials Tech Co. Ltd.

\section{Synthesis and fabrication of GHEC elastomeric gel film}

HEC ( $2 \mathrm{~g}$ ) was added to deionized water (DI water 98 $\mathrm{mL})$ at $25^{\circ} \mathrm{C}$. After stirring for $2 \mathrm{~h}$, glycerol $(0,1,2,5,10$, and $20 \mathrm{~g}$ ) was added dropwise and stirred continuously for $1 \mathrm{~h}$. Then the mixture solution was stirred for $6 \mathrm{~h}$ at $80^{\circ} \mathrm{C}$ and cooled to a set temperature, forming a GHEC aqueous solution. The typical procedure for the preparation

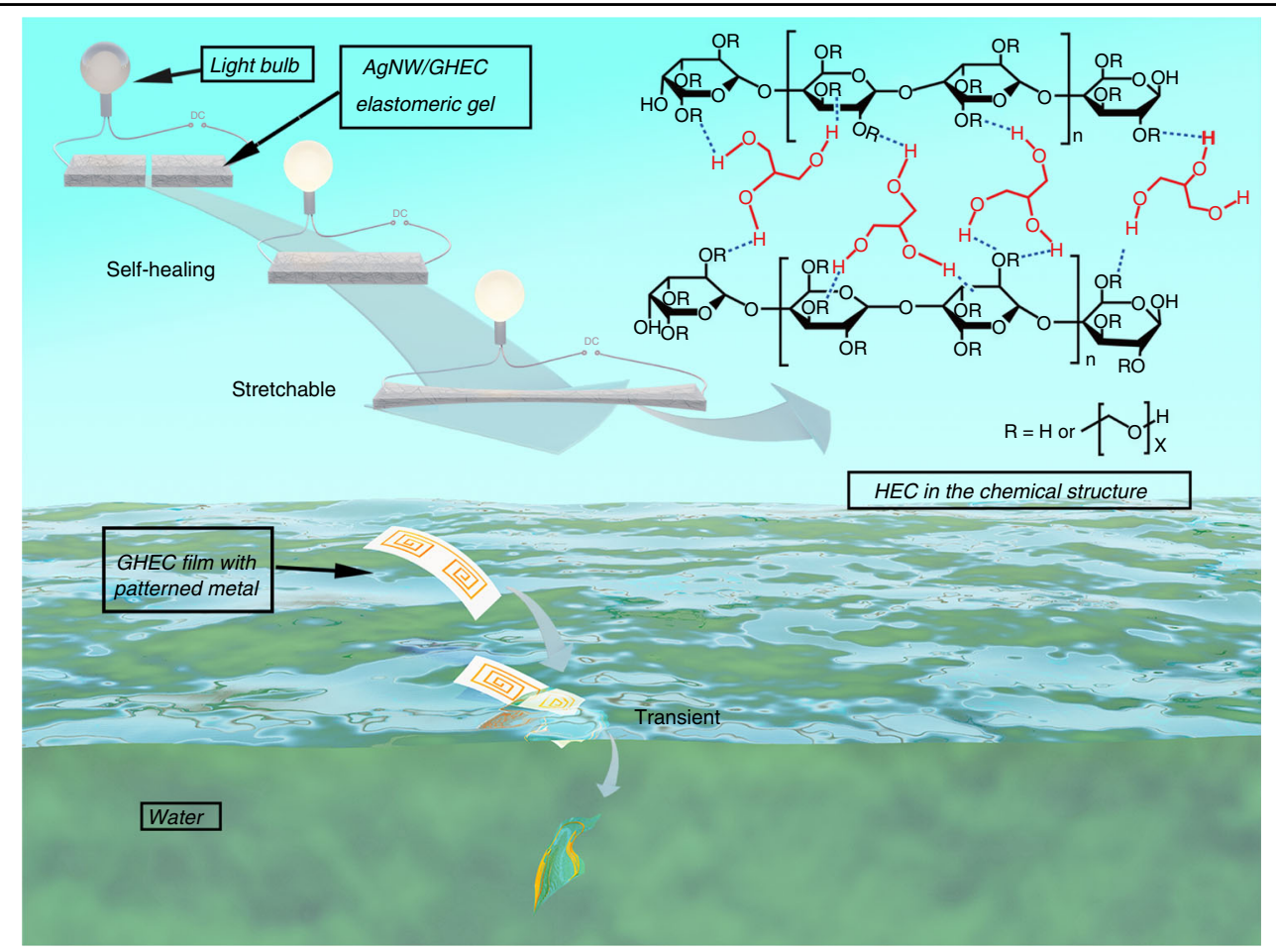

Fig. 1 Schematic of the GHEC elastomeric gel that can self-heal, stretch, and degrade 
of GHEC films is as follows: The mixture solution $(20 \mathrm{~mL})$ was poured into a quartz tank mold measuring $5 \mathrm{~cm}$ length $\times 3 \mathrm{~cm}$ width $\times 4 \mathrm{~cm}$ height and was dried at $80^{\circ} \mathrm{C}$ for $12 \mathrm{~h}$. The polymer films were then peeled off from the quartz tank mold for further testing.

\section{Preparation of the self-healing electrode}

$5 \mathrm{~mL} 0.05 \mathrm{wt} \%$ AgNWs dispersion were dispersed into the $100 \mathrm{~mL}$ GHEC aqueous solution by stirring for $4 \mathrm{~h}$. Then the mixture was poured into a quartz tank mold forming a self-healing AgNW/ GHEC elastomeric gel.

\section{Preparation of transient electronics}

The transient electronics were fabricated by patterning metal aluminum (Al) electrodes onto the GHEC films using electron beam evaporation via a shadow mask. A piece of GHEC film was first rinsed by sonication in ethanol for $10 \mathrm{~min}$ and then was blow-dried with a nitrogen gun to obtain a piece of clean degradable substrate. Then the film was treated using UV ozone for 15 min. Then the electrode was assembled using electron beam evaporation at $200^{\circ} \mathrm{C}$ for $5 \mathrm{~min}$ at a rate of $0.8-1.2 \mathrm{~nm} \mathrm{~s}^{-1}$.

\section{Preparation of multifunctional electronic skins}

The multifunctional electronic skins were fabricated by assembling a stress-sensitive layer of SWCNTs on a GHEC film. Initially the SWCNT suspension (ink) was fabricated: $2 \mathrm{mg}$ of SWCNTs ( $>98 \mathrm{wt} \%$, with an average diameter between 5 and $8 \mathrm{~nm}$, and average length between 0.5 and $5 \mu \mathrm{m}$ Nanjing XFNANO Materials Tech Co., Ltd., China) were dispersed in a $100 \mathrm{~mL}$ absolute ethyl alcohol by strong sonication $(400 \mathrm{~W})$ for $2 \mathrm{~h}$ using an ultrasonic cell disruptor (BILON 92-IIShanghai Bilon Co., Ltd). Then the SWCNT suspension (ink) was spray-coated onto the GHEC film. Finally, the coated film was dried at $80^{\circ} \mathrm{C}$ for $2 \mathrm{~h}$, forming a multifunctional electronic skin.

\section{Materials characterization and the sensor experiments}

A digital camera (Canon EOS 70D) was used to take photos and record videos. Tensile tests were performed using the Instron 3365 at a constant speed of $10 \mathrm{~mm} \mathrm{~min}^{-1}$.

\section{Dynamic mechanical analysis (DMA)}

The storage moduli and loss moduli of the elastomeric gel samples (film, $10 \mathrm{~mm}$ in length, $5 \mathrm{~mm}$ in width and $0.4 \mathrm{~mm}$ in thickness) were measured using a NETASCH DMA $242 \mathrm{E}$ with a compression mode at $10 \mathrm{~Hz}$ in the temperature range of $25-105^{\circ} \mathrm{C}$ with a heating rate of $5{ }^{\circ} \mathrm{C} \mathrm{min}{ }^{-1}$, and an amplitude of $100 \mu \mathrm{m}$. The measurement was performed in normal air atmosphere. The FTIR spectra were collected via a Thermo Nicolet iN 10 spectrometer from 4000 to $400 \mathrm{~cm}^{-1}$ at $77 \mathrm{~K}$ in a liquid nitrogen cooling environment. SEM was performed for micromorphology observation using a Hitachi S-4800 cold field emission scanning electron microscope at an accelerating voltage of $5 \mathrm{kV}$. The current measurements were performed by connecting two ends of the AgNW/ GHEC film with a digital source meter (Keithley $2602 \mathrm{~A}$ ), using two copper wires as the electrodes to record the real-time electric current $(I)$ of the film under a constant voltage $\left(V_{0}\right)$ of $2 \mathrm{~V}$.

\section{Results and discussion}

HEC is an important and abundant cellulose derivative with advantages of good biocompatibility and biodegradation properties ${ }^{27-29}$, which make it a potential candidate for multifunctional flexible and stretchable transient electronics devices. However, the bulky HEC molecular chains fold and interweave with one another due to the intermolecular forces (Van Der Waals force, including the hydrogen bond), resulting in a strong steric hindrance, limited stretchability, and approximately no self-healing ability ${ }^{30}$. To meet the requirements of multifunctional flexible and stretchable transient electronics devices with a self-healing ability, a series of supramolecular GHEC elastomeric gels were synthesized by introducing different amounts of functional guest small glycerol molecules into the HEC matrix to form new elastomeric oil gels. GHEC elastomeric gel is stable and the weight changes of the GHEC gel are less than 0.5\% under different temperatures $\left(0 \sim 90{ }^{\circ} \mathrm{C}\right)$ at both 20 and $80 \%$ RH (Figure S1).

Since hydrogen bonds are weak dynamic noncovalent bonds ${ }^{15,31}$, they will break preferentially upon damage. However, those broken hydrogen bonds can reform at the fractured interfaces due to the dynamic characteristic of supramolecular chains (Fig. 2a), which is the self-healing mechanism of the GHEC. For the insertion of small glycerol molecules, the reformation of dynamic hydrogen bonds occurs between the HEC chains and the guest small glycerol molecules (Fig. 1), which endows the HEC supramolecular chains with mobility and makes the folded HEC molecular chains easier to straighten, inducing a higher stretchability for the GHEC compared with HEC (Fig. 2b). Additionally, the GHEC elastomeric gel is watersoluble and the fundamental mechanism of dissolution can be described as follows: first, water molecules interact with the glycerol molecules at the outer layer of the GHEC. Then, the glycerol molecules between the chains of the HEC molecules are replaced by water molecules and the glycerol dissolves, which promotes the binding of water molecules to the HEC. The same process is conducted from the outer layer to the inner layer, and the GHEC is finally completely dissolved ${ }^{32,33}$. Therefore, its degradation rate can be tuned by adjusting the HEC molecular weight or the ratio of HEC to glycerol (Fig. 2c). 

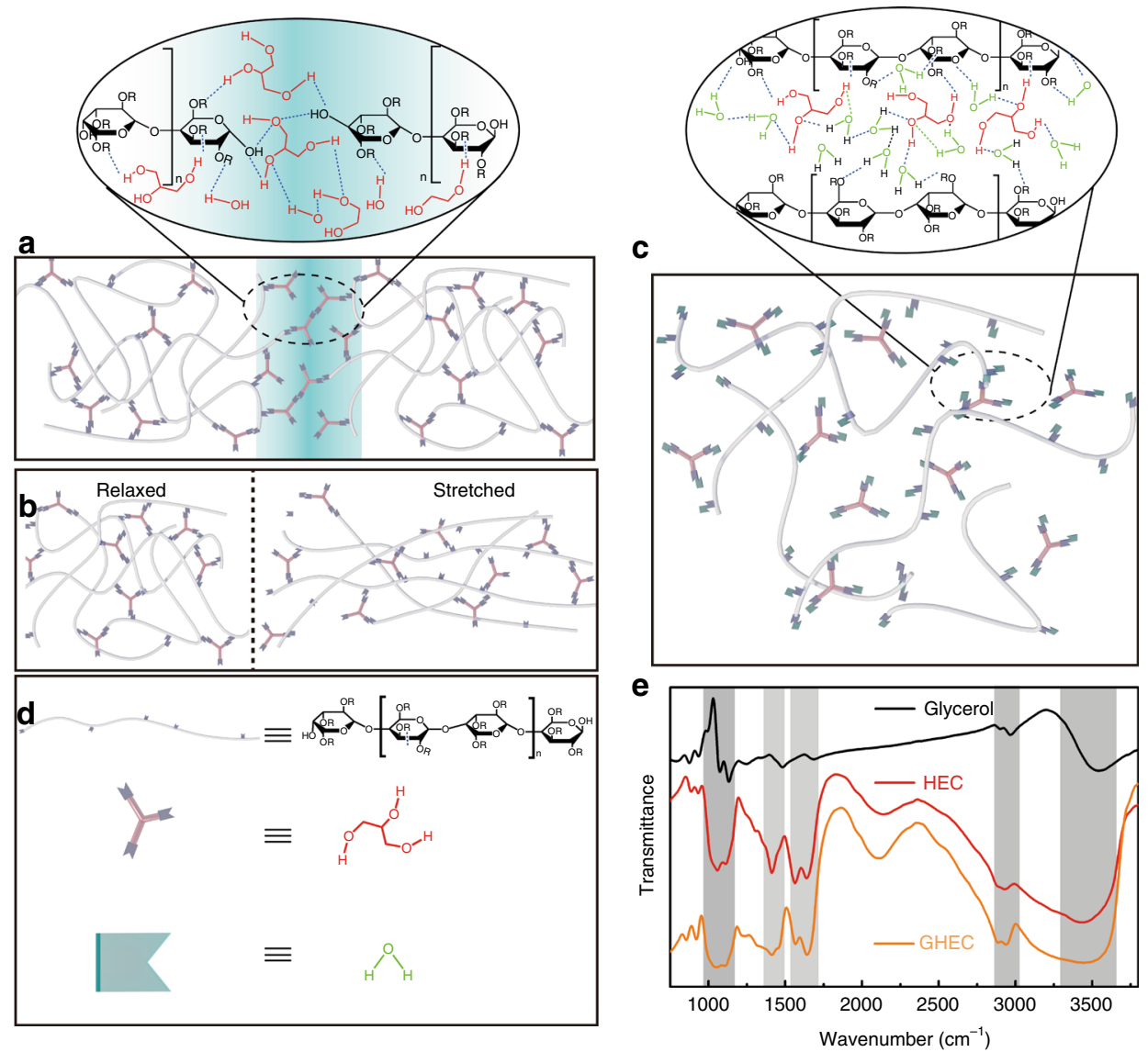

Fig. 2 Working mechanism of the GHEC elastomeric gel. a-d Schematic diagrams of the self-healing, stretching, and degrading properties of the GHEC elastomeric gel. Gray lines represent the polymer chains of HEC and form cross networks. Purple shapes on the polymer chains and brown short lines form the primary hydrogen bonds between the polymer chains and glycerol. The blue-green shapes represent water molecules. e The Fourier transform infrared spectra of various materials; the orange, black, and red lines represent glycerol, HEC and GHEC, respectively. HEC,

hydroxyethylcellulose; GHEC glycerol/hydroxyethylcellulose

To verify the molecular composition of the GHEC, the infrared spectra of the GHEC was measured. As Fig. 2e presents, the Fourier transform infrared (FTIR) spectra of the GHEC includes peaks belonging to $\mathrm{O}-\mathrm{H}$ stretching at $3500 \mathrm{~cm}^{-1}, \mathrm{C}-\mathrm{H}$ stretching at approximately 2800 $-3000 \mathrm{~cm}^{-1}$ and $\mathrm{C}-\mathrm{O}-\mathrm{C}$ stretching at approximately $1100 \mathrm{~cm}^{-1}$, which are the characteristic peaks of HEC $(\mathrm{O}$ $-\mathrm{H}, \mathrm{C}-\mathrm{H}, \mathrm{C}-\mathrm{O}-\mathrm{C})$ and glycerol $(\mathrm{O}-\mathrm{H}, \mathrm{C}-\mathrm{H})$, respectively. FTIR spectra suggests that the HEC and glycerol are simply physically mixed in the GHEC instead of crosslinking by chemical reactions. Hence, the hydroxyl functional group is not destroyed, which supports the aforementioned mechanism.

\section{Mechanical properties of GHEC elastomeric gels}

The mechanical properties of a series of GHEC elastomeric gels were characterized. As shown in Fig. 3a, the typical tensile stress-strain curves of the GHECs elastomeric gel films with various mass ratios $(m)$ of the $2 \%$
HEC2000 aqueous solution (the viscosity of HEC is $2000 \mathrm{mPa} . \mathrm{s})$ to glycerol display a decreased elasticity modulus from 25.78 to $0.02 \mathrm{MPa}$ as $m$ decreased from 1 to $5: 1$. However, the stretchability of GHEC with $m=50$ of $304 \%$ is the best, and decreased when $m$ was less than 50 since the numerous glycerol molecules form more weak hydrogen bonds between the glycerol molecules, which preferentially break before the HEC chains completely spread out ${ }^{16,32,34}$. In particular, the elasticity modulus of the GHEC with $m=20$ is approximately $0.91 \mathrm{MPa}$ and the max strain is $270 \%$, which are soft enough for wearable flexible and stretchable electronics (Fig. 3b, c). In addition to $m$, the HEC molecular polymerization degree is another key factor that affects the mechanical properties of the GHEC elastomeric gel. As shown in Figure S2a and b, the GHEC films with larger viscosity HEC molecules exhibit stronger elasticity moduli and worse stretchability, which are attributed to the larger molecule chains inducing a greater steric hindrance. 


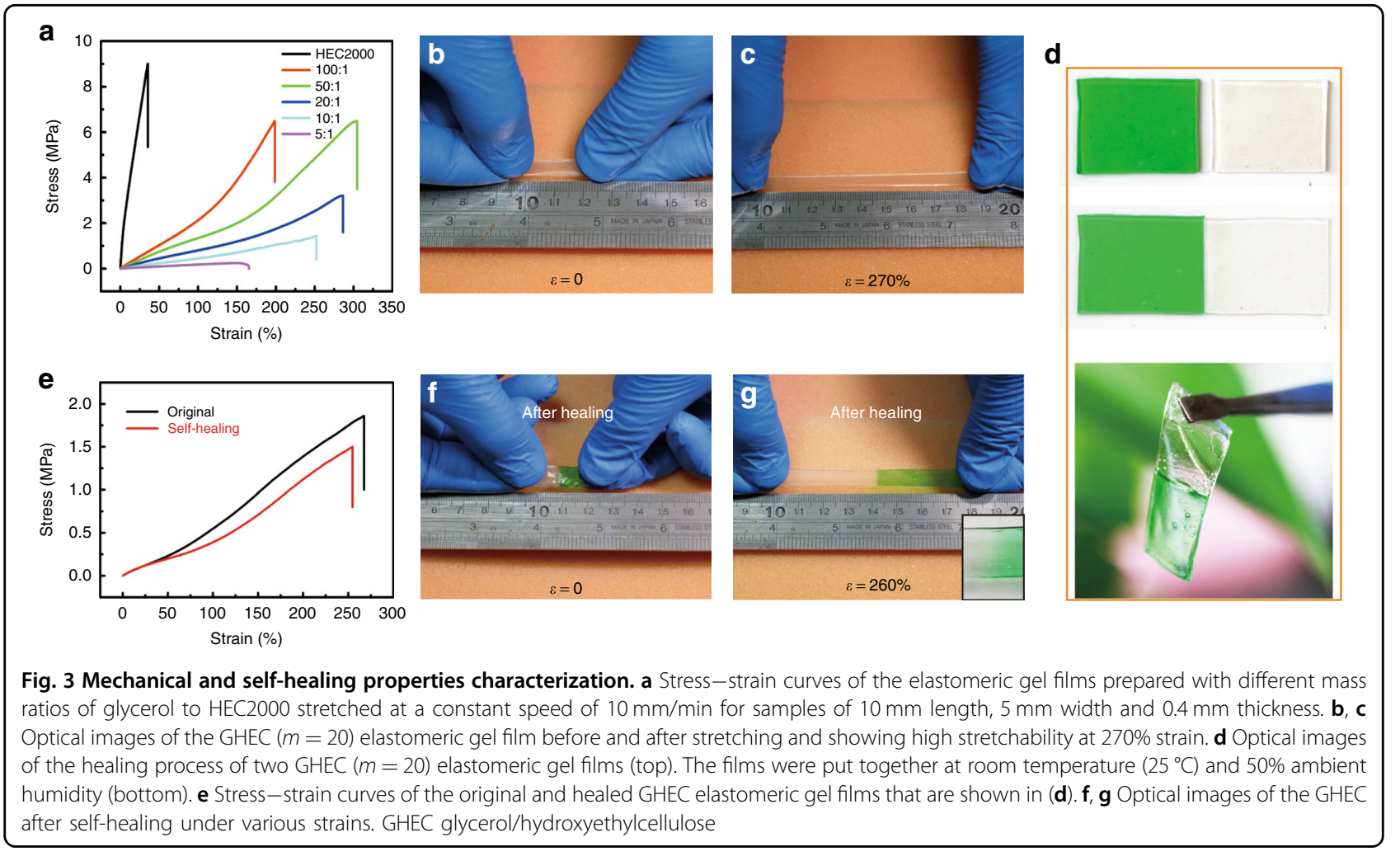

\section{Characterization of the self-healing ability of the GHEC}

For the presence of dynamic hydrogen bonds in the GHEC elastomeric gel, the reversible formation of the hydrogen bonds provides the GHEC with a preeminent selfhealing ability, which is proven by the healing process shown in Fig. 3d. The mechanical properties are one of the most effective ways to evaluate the material's healing efficiency, because they take the restoration of both the stress and strain into account. We define the mechanical healing efficiency $\eta_{\mathrm{m}}$ as the ratio of the restored maximum strain to the original maximum strain. To investigate the healing performance of GHEC2000, the stress-strain curves of a GHEC2000 film before and after healing were measured. As presented in Fig. 3e, after healing for $8 \mathrm{~min}$ in ambient humidity $(50 \% \mathrm{RH})$ and temperature $\left(25^{\circ} \mathrm{C}\right), \eta_{\mathrm{m}}$ reaches up to $96.3 \%$, which is further demonstrated by the optical pictures (Fig. $3 \mathrm{f}-\mathrm{g}$, Movie 1). The interface of the two fractures (the green and colorless parts) after healing is hazy, as shown in the inset of Fig. 3g, proving that the healing process is a typical supramolecular assembly with dynamic hydrogen bonds.

To further understand the self-healing properties of the GHEC elastomeric gel system, the self-healing processes under various environmental conditions were investigated. Figure $4 \mathrm{a}$ depicts the maximum strain of the GHEC2000 elastomeric gel films with $m=20$ during the self-healing process under various ambient humidities from 10 to $80 \% \mathrm{RH}$ at a constant temperature of $25^{\circ} \mathrm{C}$. As illustrated, it only takes $9 \mathrm{~min}$ to achieve $100 \%$ mechanical repair under $80 \% \mathrm{RH}$ and $15 \mathrm{~min}$ to achieve the same healing level under $10 \% \mathrm{RH}$, which confirms that the restoration behavior of the GHEC has no obvious requirement for humidity but that a higher humidity can effectively accelerate the self-healing speed. These results can be interpreted as being due to the water molecules improving the HEC chain's dynamic and assembly activities, thereby quickening the healing process. A kinetically increasing temperature can also increase the dynamic activity of the molecule chains, and thus the influence of temperature on the self-healing properties of the GHEC deserves to be studied. As seen from Fig. 4b, the healing speed of the GHEC2000 elastomeric gel films indeed increases as the ambient temperature increases from 0 to $80^{\circ} \mathrm{C}$ when the ambient humidity was stable at $50 \% \mathrm{RH}$. Interestingly, the healing time required to achieve a $100 \%$ restoration efficiency at room temperature, $25^{\circ} \mathrm{C}$, is only $11 \mathrm{~min}$. However, $15 \mathrm{~min}$ is required to attain $10 \% \eta_{\mathrm{m}}$. The self-healing speed plummets when the temperature reaches $0{ }^{\circ} \mathrm{C}$, which is attributed to the water molecules crystallizing from the liquid to the solid state, which greatly hinders the molecule chain dynamics and the assembly activities, thus severely limiting the repair speed. Moreover, a dynamic mechanical analysis (DMA) was conducted to inspect the dependence of the moduli of the GHEC (both before and after self-healing) on the temperature because the mechanical properties of the GHEC 

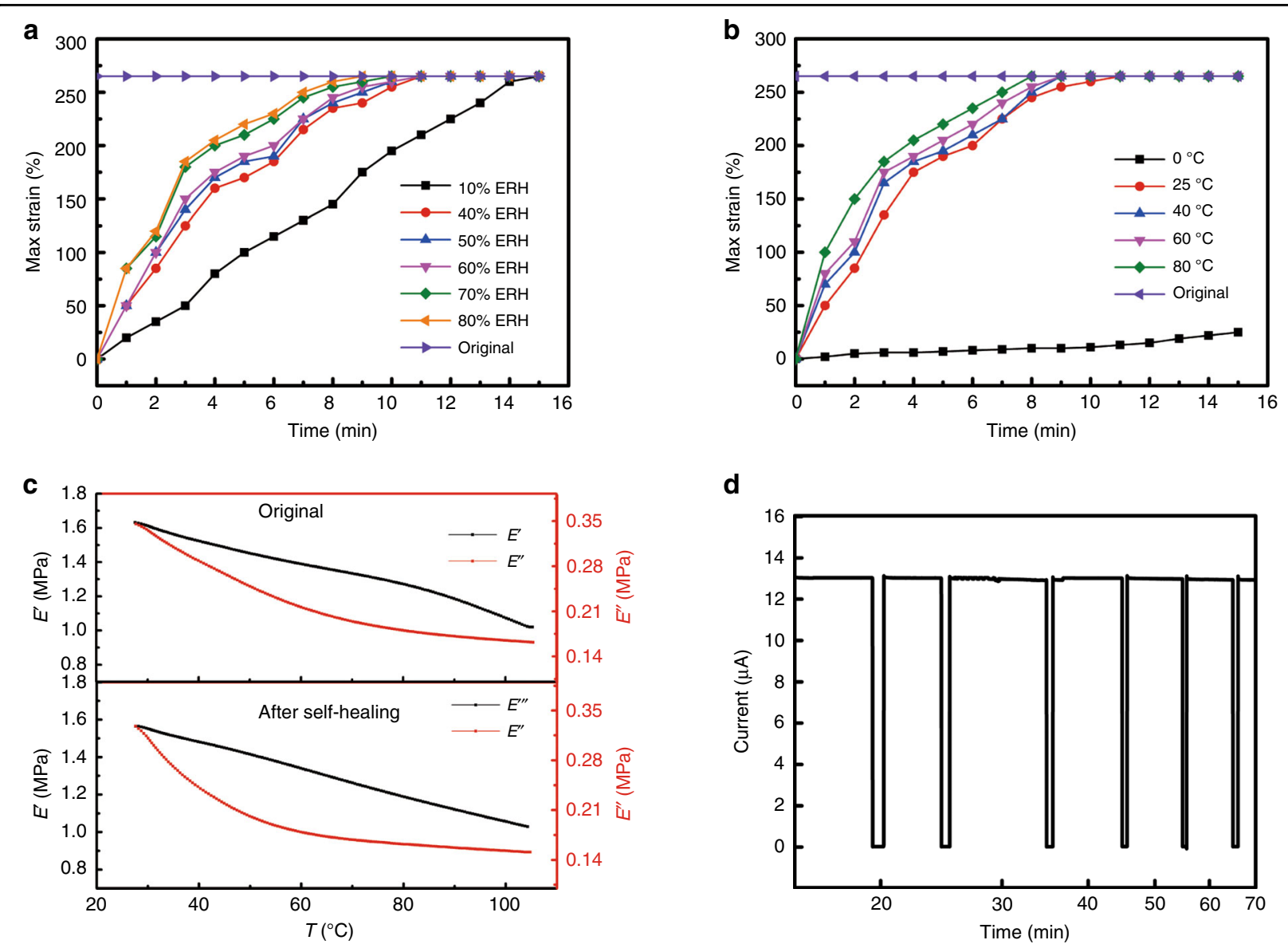

d
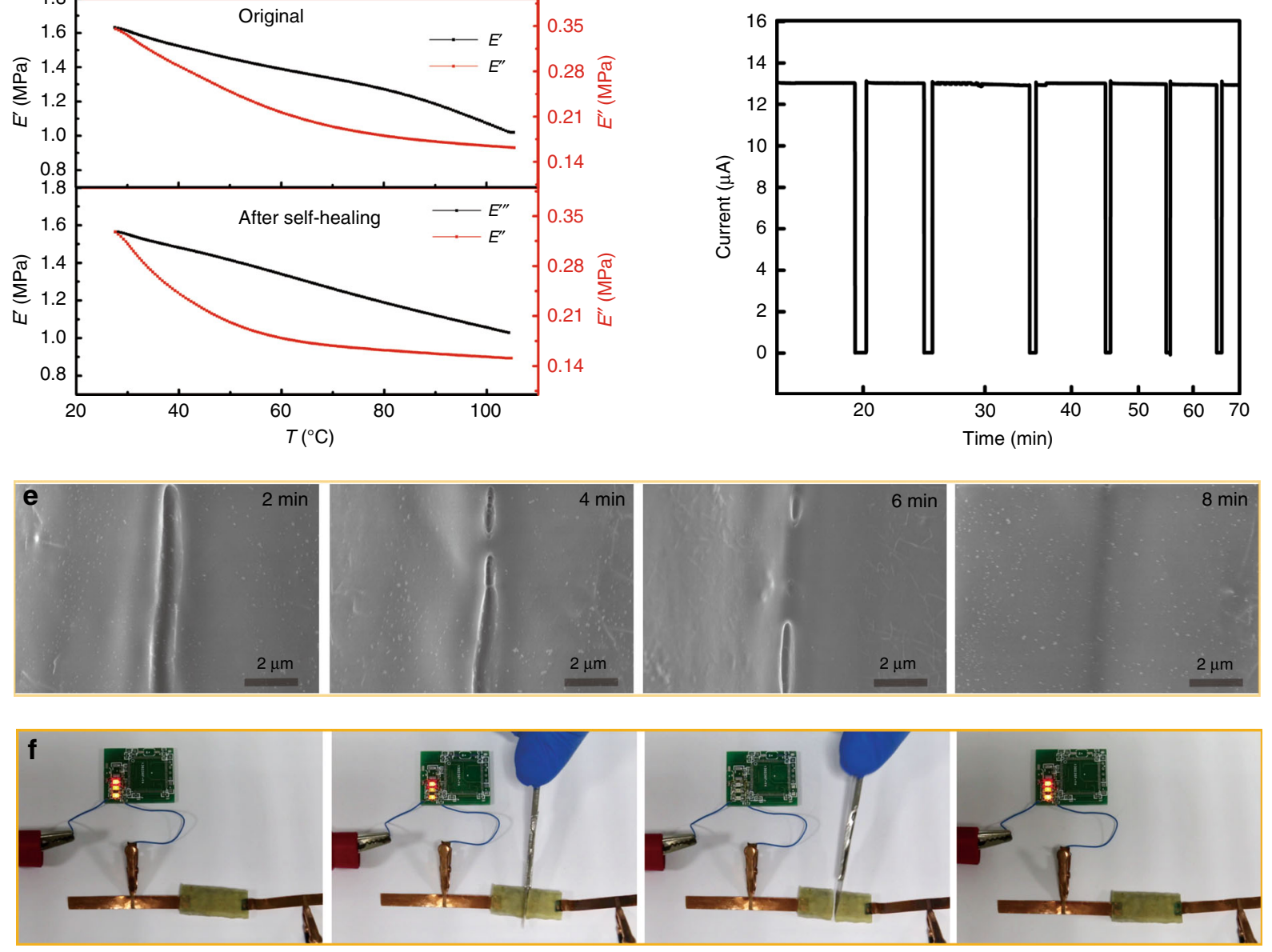

Fig. 4 The main influencing factors and the electricity self-healing property characterization. a The maximum strain-time curves of the GHEC films healed under various ambient humidities at constant room temperature $\left(25^{\circ} \mathrm{C}\right)$. b The maximum strain-time curves of the GHEC films healed at various ambient temperatures under a constant ambient humidity (50\%). c The dynamic mechanical analysis results of the GHEC $(m=20)$ elastomeric gel films. Variations of the storage moduli and loss moduli before and after self-healing as a function of the temperature from 25 to $105^{\circ} \mathrm{C}$. d Repeated electrical healing for six cuts at the same severed location by the current-time curve at room temperature. e SEM microscope images showing the self-healing process of AgNW/GHEC. $\mathbf{f}$ Optical images of a battery-powered circuit to further prove the potential of electromechanical self-healing for electronic circuits. GHEC glycerol/hydroxyethylcellulose

elastomeric gel can greatly affect the performance of flexible wearable electronics. Figure 4c shows the variation in the storage moduli $\left(E^{\prime}\right)$ and loss moduli $\left(E^{\prime \prime}\right)$ of the representative GHEC elastomeric gel (up: original; down: after self-healing) as a function of the temperature over a range from 30 to $105^{\circ} \mathrm{C}$. Apparently both the $E^{\prime}$ and $E^{\prime \prime}$ 
values of the GHEC elastomeric gel before and after selfhealing drop rapidly with an increase in the temperature from 30 to $105^{\circ} \mathrm{C}$, which suggests that the GHEC elastomeric gel becomes weak and softens with an increase in the temperature due to the dynamic breakage of intermolecular hydrogen bonds between the glycerol and HEC. Additionally, the DMA curves of the GHEC elastomeric gel before and after self-healing have no obvious attenuation, demonstrating the excellent mechanical selfhealing ability.

In addition to the mechanical properties, the electrical property restoration is another valuable way to characterize the self-healing ability of the GHEC. Figure S3 shows an SEM image of an AgNW/GHEC elastomeric gel film produced by casting a uniformly dispersed silver nanowire (AgNWs) GHEC aqueous solution. Such an AgNW/GHEC elastomeric gel displays a good electrical conductivity due to the formation of AgNWs networks in the elastomeric gel matrix. To investigate the electrical healing, the AgNW/GHEC elastomeric gel film was completely bifurcated using a scalpel and the two fractured surfaces were brought together. A digital source meter (Keithley 2602A) was used to record the real-time current with a constant applied voltage of $1 \mathrm{~V}$ DC. As illustrated in Fig. 4d, once bifurcated the current immediately goes to 0 , forming an open circuit. As the edges of the elastomeric gel were brought into contact, the conductivity increased, and the current returned to the initial value within $1 \mathrm{~s}$. The elastomeric gel became selfsupporting after $30 \mathrm{~s}$, demonstrating the excellent repeatable restoration of the electrical performance. The series of SEM images in Fig. 4e exhibits details of the AgNW/ GHEC elastomeric gel film healing process. Clearly the AgNWs at the edges of the elastomeric gel can immediately contact one another, forming new conductive networks, which results in the fast restoration of the electrical performance. Subsequently, supermolecular chains diffuse, cross-link with one another, gradually begin to be self-supporting, and eventually achieve complete mechanical properties restoration. Finally, a batterypowered circuit was constructed to further prove the potential of electromechanical self-healing for electronic circuits (Fig. 4f, Movie 2). The LED light in the series circuit are extinguished once cut off from the self-healing conductive elastomeric gel and then were lit again after transitory healing. Electrical and mechanical performances during ten instances of failures and recoveries (cutting-self-healing cycles) were characterized to further demonstrate the stability. As shown in Figure S4, the maximum strain of AgNW/GHEC decreases 5\% and the resistance increases $0.46 \%$ after ten instances of failures and recoveries, verifying the repeatable restoration of the electrical and mechanical performances.

\section{Degradability characterization of GHEC elastomeric gel}

Sensitive data leakage prevention, the reduction of electronic waste, and biomedical implants without second surgery are urgent requirements for controllable transient electronics. The GHEC elastomeric gel system is watersoluble and the degradation rate can be tuned by adjusting the HEC molecular weight or the ratio of HEC to glycerol, which makes GHEC a promising transient electronic substrate. Figure 5a shows a 1-mm-thick GHEC2000 $(m=20)$ film with patterned metal aluminum (Al) electrodes that was prepared using electron beam evaporation. Optical photographs (Figure 5b-f) detail the GHEC film degradation process after immersion in $25^{\circ} \mathrm{C}$ water: The GHEC substrate absorbs water and begins to swell within $2 \mathrm{~min}$. Meanwhile, the electrode falls off the HEC substrate and disintegrate into small fragments, which represents the loss of structure and electrical performance of the flexible electrodes system. After $20 \mathrm{~min}$ of degradation, all the electrodes disappear, and the substrate becomes a fluid flocculation, which proves that GHEC can be used as a rapidly transient electronic device. To further investigate the degradation property of the GHEC, a series of GHEC

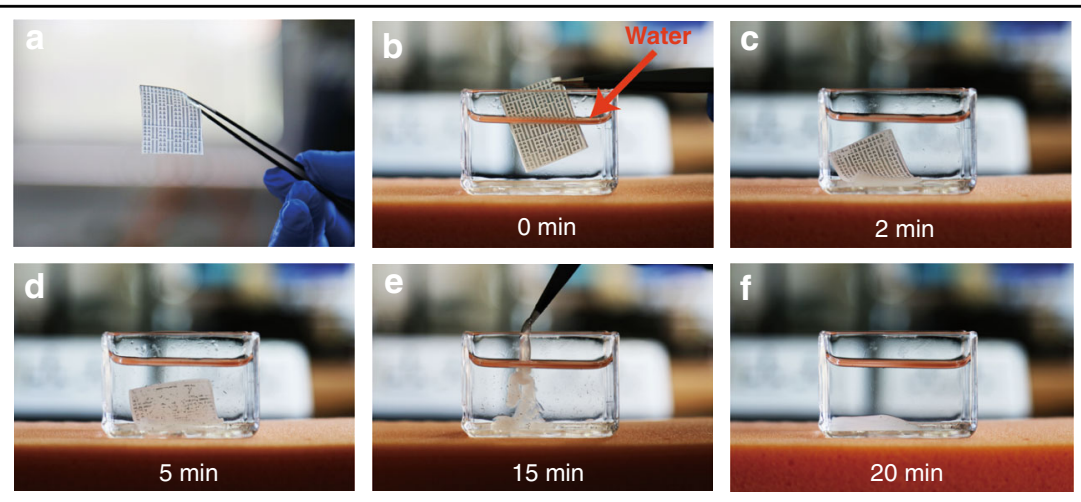

Fig. 5 transience process of the GHEC film with patterned metal aluminum (Al) electrodes. a-f Optical images of the transience process of the GHEC film with patterned metal aluminum (Al) electrodes during various degradation stages. GHEC glycerol/hydroxyethylcellulose 


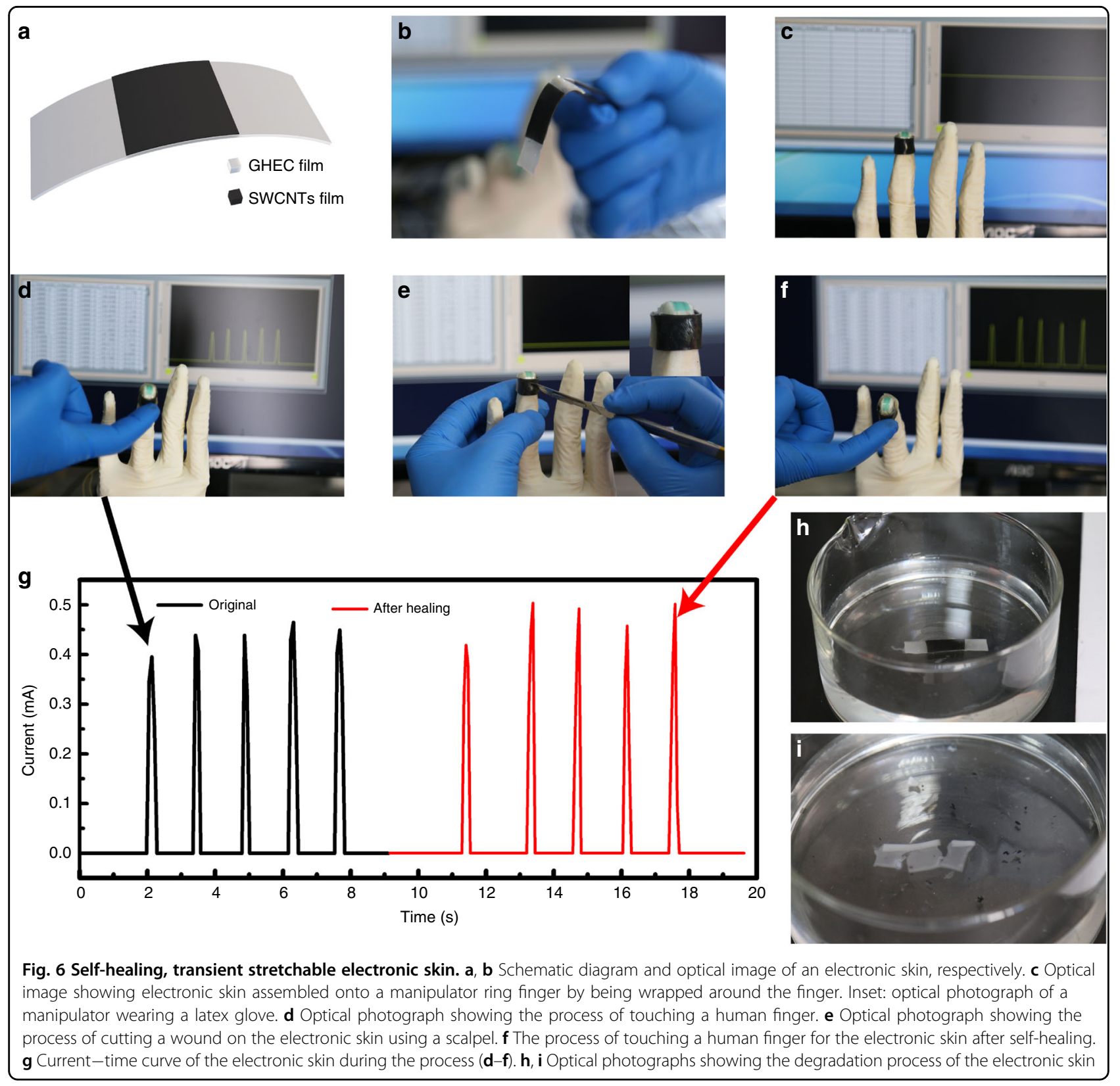

with different components were fabricated. As shown in Figure S5, the degradation rate of the GHECs $(m=20)$ with a low viscosity of HEC is obviously faster than that of the GHECs with a high viscosity of HEC, which is caused by the fact that short chains have less intermolecular interaction sites $^{32}$. Moreover, as the increase in glycerol can also effectively decrease the HEC intermolecular interaction sites, promoting the GHEC degradation rate, it therefore takes a shorter time for the GHECs with a small $m$ to degrade (for example, the degradation time is approximately 60 min for GHEC with $m=20$, while the degradation time is more than $90 \mathrm{~min}$ for that with $m=50$, shown in Figure S6). The ambient temperature is also an important factor that influences the degradation rate, and a high temperature can accelerate the degradation rate of the thermal motion of molecules (Figure S7). Additionally, the electrical properties of the material do not change along with the mass ratios of glycerol and HEC or the viscosities of HEC, because GHEC is a nonionic mixture of glycerol and HEC, which has weak electrical conductivity in ambient conditions. The resistance of GHEC gel $(m=20)$ film decreases from 8 to $3 \mathrm{M} \Omega$ after immersion in water, which is greater than AgNW/GHEC (75 k $\Omega$ ), shown in Figure S8. Assembling conductive materials (such as AgNWs, CNTs, etc.) in or on a GHEC film is the main way to endow GHEC gel with conductivity. 


\section{Demonstration of electronic skin based on the self-healing transient stretchable GHEC elastomeric gel}

Finally, a practical application of the self-healing transient stretchable GHEC was demonstrated by assembling a stress-sensitive layer of SWCNTs on a multifunctional GHEC film to form an electronic skin (Fig. 6a), which is widely used in wearable electronics and human-machine interfaces. Figure $6 \mathrm{~b}$ shows a piece of electronic skin (see Methods for the fabrication method), which can be assembled onto the curved surface of a manipulator ring finger by being wrapped around the finger, since the crosssection of the electronic skin automatically heals under environmental conditions (Fig. 6c). Figure 6d and Movie 3 (Supporting Information) present the real-time pressure detection of the ring finger. As the finger bends, driven by the controller, to touch the object repeatedly, its current shows a periodic synchronous increase and decrease, achieving real-time pressure sensing, which is very important for creating intelligent robot perception and human -computer interaction. The real-time current of the electronic skin was measured using a digital source meter (Keithley 2602A) under a given voltage of $1 \mathrm{~V}$. Moreover, to simulate a real mechanical injury, we used a scalpel to cut a wound on the electronic skin (Fig. 6e and Movie 3). After a short period for self-healing, the manipulator ring finger regains its sensing ability, which is approximately identical to its previous sensing performance, as shown in Fig. 6f, $g$ (the slight difference between each cycle resulted from the slight discrepancy of each bend), indicating a good selfhealing ability to resist mechanical damage. The stability of the electronic skin is further proven by ten cycles of repeated failures and recoveries (Figure S9). Finally, the electronic skin was removed and immersed in water for degradation (Fig. 6h, i). The multifunctional electronic skin demonstrates the abilities of stretchability, self-healing and degradation of the GHEC, which extend the flexible wearable electronic applications through the prevention of sensitive data leakage, the reduction of electronic waste, and allowing biomedical implants without a second surgery.

\section{Conclusions}

In summary, a series of stretchable macromolecular elastomeric gels with both self-healing and degradation abilities under room temperature were developed. Based on this, a series of electronics devices were demonstrated that can work after wound healing and then degrade controllably. By inserting small molecule glycerol into HEC, forming a GHEC elastomeric gel system, dynamic hydrogen bonds occur between the HEC chain and the guest small glycerol molecules, which endows the GHEC with an excellent stretchability and a self-healing ability under normal environment conditions. Additionally, the GHEC elastomeric gel system is water-soluble and its degradation rate can be tuned by adjusting the HEC molecular weight or the ratio of HEC to glycerol. The multifunctional GHEC, which simultaneously has the abilities of self-healing to resist mechanical damage, maintains a stable performance for a desired amount of time and completely disappears under controlled programs, extends the flexible wearable electronic applications through the prevention of sensitive data leakage, the reduction of electronic waste, and allowing biomedical implants without a second surgery.

\section{Acknowledgements}

The authors acknowledge the funding support from the National Key R\&D Program of China (2017YFA0701101), the National Natural Science Foundation of China $(61574163,61801473)$, the Science Foundation for Distinguished Young Scholars of Jiangsu Province, China (BK20170008), and the NANO-X Workstation scientifically supported this research.

\section{Conflict of interest}

The authors declare that they have no conflict of interest.

Supplementary information accompanies this paper at https://doi.org/ 10.1038/s41378-019-0047-4.

Received: 5 November 2018 Revised: 20 December 2018 Accepted: 28 December 2018

Published online: 11 March 2019

\section{References}

1. Li, L. H. et al. Coating for flexible and wearable sensing electronics. Adv. Mater. 29, 1702517 (2017)

2. Li, L. H. et al. Ultrastretchable fiber sensor with high sensitivity in whole workable range for wearable electronics and implantable medicine. Adv. Sci. $\mathbf{5}$, 1800558 (2018).

3. Trung, T. Q. \& Lee, N. E. Flexible and stretchable physical sensor integrated platforms for wearable human-activity monitoring and personal healthcare. Adv. Mater. 28, 4338-4372 (2016)

4. Dagdeviren, C. et al. Conformable amplified lead zirconate titanate sensors with enhanced piezoelectric response for cutaneous pressure monitoring. Nat. Commun. 5, 4494 (2014)

5. Park, J. et al. Tactile-direction-sensitive and stretchable electronic skins bsed on human-skin-inspired interlocked microstructures. Acs Nano 8, 12020-12029 (2014).

6. Lin, Z. M. et al. Triboelectric nanogenerator enabled body sensor network for self-powered human heart-rate monitoring. Acs Nano 11, 8830-8837 (2017).

7. Schwartz, G. et al. Flexible polymer transistors with high pressure sensitivity for application in electronic skin and health monitoring. Nat. Commun. 4, 1859 (2013).

8. Kim, D. H. et al. Materials for multifunctional balloon catheters with capabilities in cardiac electrophysiological mapping and ablation therapy. Nat. Mater. 10, 316-323 (2011).

9. Hattori, Y. et al. Multifunctional skin-like electronics for quantitative, clinical monitoring of cutaneous wound healing. Adv. Healthc. Mater. 3, 1597-1607 (2014).

10. Lim, S. et al. Transparent and stretchable interactive human machine interface based on patterned graphene heterostructures. Adv. Funct. Mater. 25, 375-383 (2015).

11. Son, D. et al. Multifunctional wearable devices for diagnosis and therapy of movement disorders. Nat. Nanotechnol. 9, 397-404 (2014).

12. Jung, $\mathrm{S}$. et al. Reverse-micelle-induced porous pressure-sensitive rubber for wearable human-machine interfaces. Adv. Mater. 26, 4825-+ (2014).

13. Lee, S. et al. A strain-absorbing design for tissue-machine interfaces using a tunable adhesive gel. Nat. Commun. 5, 5898 (2014). 
14. Wang, $X$. et al. Dynamic pressure mapping of personalized handwriting by a flexible sensor matrix based on the mechanoluminescence process. Adv. Mater. 27, 2324-2331 (2015).

15. Chen, D. et al. Self-healing materials for next-generation energy harvesting and storage devices. Adv. Energy Mater. 7, 1700890 (2017).

16. Yan, X. et al. Quadruple H-bonding cross-linked supramolecular polymeric materials as substrates for stretchable, antitearing, and self-healable thin film electrodes. J. Am. Chem. Soc. 140, 5280-5289 (2018).

17. Tee, B. C. K., Wang, C., Allen, R. \& Bao, Z. N. An electrically and mechanically selfhealing composite with pressure- and flexion-sensitive properties for electronic skin applications. Nat. Nanotechnol. 7, 825-832 (2012).

18. Markvicka, E. J., Bartlett, M. D., Huang, X. \& Majidi, C. An autonomously electrically self-healing liquid metal-elastomer composite for robust soft-matter robotics and electronics. Nat. Mater. 17, 618-624 (2018).

19. Lai, Y. et al. Colorless, transparent, robust, and fast scratch-self-healing elastomers via a phase-locked dynamic bonds design. Adv. Mater. 30, e1802556 (2018).

20. Cordier, P., Tournilhac, F., Soulie-Ziakovic, C. \& Leibler, L. Self-healing and thermoreversible rubber from supramolecular assembly. Nature 451, 977-980 (2008).

21. Huang, $X$. et al. Biodegradable materials for multilayer transient printed circuit boards. Adv. Mater. 26, 7371-7377 (2014).

22. Park, C. W. et al. Thermally triggered degradation of transient electronic devices. Adv. Mater. 27, 3783-3788 (2015).

23. Hwang, S. W. et al. A physically transient form of silicon electronics. Science 337, 1640-1644 (2012).

24. Gao, Y. et al. Moisture-triggered physically transient electronics. Sci. Adv. 3, e1701222 (2017).

25. Boutry, C. M. et al. A stretchable and biodegradable strain and pressure sensor for orthopaedic application. Nat. Electron. 1, 314-321 (2018)
26. Hwang, S. W. et al. High-performance biodegradable/transient electronics on biodegradable polymers. Adv. Mater. 26, 3905-3911 (2014)

27. Zhang, J. et al. Patterned arrays of supramolecular microcapsules. Adv. Funct. Mater. 28, 1800550 (2018).

28. Gast, A. P., Russel, W. B. \& Hall, C. K. An experimental and theoretical-study of phase-transitions in the polystyrene latex and hydroxyethylcellulose system. J. Colloid Interf. Sci. 109, 161-171 (1986).

29. Svensson, A. V., Huang, L. G., Johnson, E. S., Nylander, T. \& Piculell, L. Surface deposition and phase behavior of oppositely charged polyion/surfactant ion complexes. 1. Cationic guar versus cationic hydroxyethylcellulose in mixtures with anionic surfactants. Acs Appl. Mater. Inter. 1, 2431-2442 (2009).

30. Kono, $\mathrm{H}$. et al. CP/MAS C-13 NMR study of cellulose and cellulose derivatives. 1 . Complete assignment of the CP/MAS C-13 NMR spectrum of the native cellulose. J. Am. Chem. Soc. 124, 7506-7511 (2002).

31. Du, R. et al. Hierarchical hydrogen bonds directed multi-functional carbon nanotube-based supramolecular hydrogels. Small 10, 1387-1393 (2014).

32. Patruyo, L. G., Muller, A. J. \& Saez, A. E. Shear and extensional rheology of solutions of modified hydroxyethyl celluloses and sodium dodecyl sulfate. Polym. (Guildf.). 43, 6481-6493 (2002).

33. Ingham, J. D. \& Rapp, N. S. Polymer degradation .2. Mechanism of thermal degradation of polyoxypropylene glycol-toluene 2,4-diisocyanate polymer (Popg-Tdi)+block polyether glycol-Tdi polymer. J. Polym. Sci. Part A 2(11p), 4941- (1964).

34. Rong, Q. F. et al. Anti-freezing, conductive self-healing organohydrogels with stable strain-sensitivity at subzero temperatures. Angew. Chem. Int. Ed. 56, 14159-14163 (2017). 